\title{
Associative learning in plants: light quality history may matter
}

\author{
Fatima CVRČKOVÁ*; Hana KONRÁdOVÁ \\ Department of Experimental Plant Biology, Faculty of Science, Charles University, Prague, CZ 128 43, Czech Republic
}

Key words: Classical conditioning, Light quality, Phototropism, Replicability, Reproducibility

\begin{abstract}
The possibility of associative learning in plants is a topic of ongoing controversy. In one published study, growing pea plants were reported to associate two stimuli (airflow and light) and thereafter use one (airflow) as an indicator for the other (light), similar to dogs in Pavlov's famous experiments. However, this observation could not be independently repeated. Here we examine a possible reason for the failure of a published reproduction attempt, which used substantially different light quality during plant cultivation prior to experimental treatments than in the original study. This could have resulted in dramatically different growth characteristics. While the relevance of the original report of plant associative learning remains questionable, greater attention should be paid to good documenting and standardizing the light conditions, in particular spectral quality, not only in studies of plant learning and memory, but also in other areas of experimental plant biology.
\end{abstract}

Introduction: Associative Learning Does Not Require a Brain

Five years ago, a study documenting classical conditioning in plants (Gagliano et al., 2016) gained considerable attention (Rasmussen, 2018; Burgos, 2018; Calvo et al., 2020; Mallatt et al., 2021). However, a subsequent attempt to replicate the published experiments failed (Markel, 2020a). Thus, the possibility of associative learning in plants remains a subject of debate, despite efforts to find causes of the apparent lack of reproducibility of the originally reported results (Gagliano et al., 2020; Markel, 2020b).

Obviously, the experimental studies that started the ongoing controversy need to be critically examined. Cognitive and behavioral scientists have readily embraced the notion that learning can take place in the absence of consciousness, and even of a nervous system of the kind known from metazoans (summarized, e.g., in Rasmussen, 2018; Burgos, 2018). Experimental observations suggest the possibility of some form of associative learning in bacterial and yeast populations (Mitchell et al., 2009; for a critical reassessment see Lyon, 2015) as well as in protozoans (de la Fuente et al., 2019; Gershman et al., 2021). Formal models predict the possibility of associative learning in unicellular organisms through reinforcement of regulatory pathways in biochemical and genetic networks, which may be modelled

*Address correspondence to: Fatima Cvrčková,

fatima.cvrckova@natur.cuni.cz

Received: 29 June 2021; Accepted: 02 September 2021 as neural networks (Tagkopoulos et al., 2008; Fernando et al., 2009). In principle, any genetic regulatory network comprising bistable elements can form and store associations (Sorek et al., 2013). Since such networks abound in any type of cells, the possibility of associative learning in plants is theoretically possible, although this does not mean that it has to exist.

\section{Examples of Perception and Memory in Plants}

Plants sense many environmental variables (e.g., the quantity, quality and photoperiod of light, temperature, humidity, $\mathrm{CO}_{2}$ level, soil moisture, and nutritional status), and integrate their sensory outputs through complex signaling networks (Paik and Huq, 2019). It is thus not surprising that plants evolved mechanisms to remember past events and prime their responses in order to react faster or stronger to recurrent environmental change, i.e., learn from past experiences. Examples of both short-term and long-term memory formation, which is a prerequisite of (if not synonymous with) learning, have been documented in plants (for reviews see, e.g., Cvrčková et al., 2009; Abramson and ChicasMosier, 2016; Crisp et al., 2016). Some cases of plant memory, such as vernalization or abiotic stress priming (Bouché et al., 2017; Oberkofler et al., 2021; Bhadouriya et al., 2021), involve known molecular mechanisms of epigenetic regulation of gene expression.

Light-related responses deserve special attention, because light plays a central part in plant life, providing not only energy for photosynthesis, but also information that triggers 
reactions to environmental changes. Examples such as developmental responses to sun and shade (Ballaré and Pierik, 2017) can be understood as relatively simple modifications of physiological processes by environmental inputs. However, in some cases, responses to light may involve memory formation or recall. For example, circumnutation-the periodic, typically circular movement of plant shoots, driven at least in part endogenously and often exhibiting an ultradian period (Stolarz, 2009)-may enable plants to perceive and record directional variation in light intensity by relating light exposure to specific phases of the circumnutation cycle. The growing apex can thereby generate an internal model of light distribution and grow towards the light source even in the absence of specialized organs for directional light sensing (Trewavas, 2017), analogous to a model recently proposed for orientation of roots in a heterogeneous environment (Loshchilov et al., 2021).

Thus, the ability of plants to store and use information about past events, i.e., to memorize and recall, is a welldocumented phenomenon.

\section{Plant Associative Learning: A Problem of Replicability}

Historical attempts to detect associative learning in plants have focused mainly on short-term responses, such as mechanically induced folding of Mimosa pudica leaflets, and produced inconclusive results (reviewed in Adelman (2018). Experimental evidence for associative learning in plants was, however, reported in a recent study (Gagliano et al., 2016) exploring longer term growth phenomena. In the experiments of Gagliano et al. (2016), young pea (Pisum sativum) plants, pre-grown in an illuminated controlled environment chamber and subsequently kept in the dark with brief repeated "training" exposures to blue light coming from changing directions, were found to orient their growth with respect to airflow from a fan, administered from either the same or opposite direction as light during the training. Control plants, exposed only to directional light but no airflow, oriented their growing apices toward the last light position. Thus, brief directional illumination could be considered as an unconditioned stimulus, while airflow served as a conditioned stimulus, utilized by the plants to anticipate the position of next light pulse.

However, in a repetition of these experiments (Markel, 2020a), the plants' ability to remember the direction of last light exposure and grow consistently towards it in the absence of airflow, as reported by Gagliano et al. (2016), was not reproduced. Some of the growing apices oriented in a random direction instead, possibly related to their vigorous circumnutation (Markel, 2020b)-i.e., the unconditioned stimulus did not work in the reproduction attempt as reliably as it did in the original study.

There are several obvious possible causes of the reported non-reproducibility of the Gagliano et al. (2016) results, reflected in the subsequent published discussion (Gagliano et al., 2020; Markel, 2020a, 2020b). The plant's ability to remember past light direction appears to be rather sensitive to environmental variations. For example, in the original study, training had to take place at specific phases of the circadian cycle to achieve the "learned" outcome (Gagliano et al., 2016; Gagliano et al., 2020). The original study
(Gagliano et al., 2016) and the subsequent failed repetition (Markel, 2020a) also differed in other aspects as discussed below; thus, the Markel (2020a) report should be considered an attempt at reproduction (with substantial variation to the experimental material and procedures) rather than replication in an identical setup (Plesser, 2018).

First, different pea cultivars have been used because of unavailability of the original Australian variety in the USA, and the soil composition may not have been identical. While there are reasons to believe that this is unlikely to be the cause of observed discrepancies, this possibility cannot be entirely dismissed especially concerning the genotype difference between the cultivars (Markel, 2020a). For example, even subtle differences in diurnal starch and sucrose turnover (Stitt and Zeeman, 2012) could have translated into noticeable changes in growth and/or in light responses of the two pea varieties under near-continuous light starvation. Notably, the two studies used plants of different chronological ages (about 3-4 days in Gagliano et al. (2016) vs. 5-8 days in Markel (2020a), suggesting different seedling growth characteristics. In other cultured plants, quite dramatic responses to light signals could be traced to a single mutational event, as documented, e.g., for continuous light sensitivity in the tomato (Velez-Ramirez et al., 2014); single mutations thus could be responsible for major differences in the responses of two otherwise similar plant varieties.

Furthermore, there could also have been some differences in the light conditions during the experiments, with the Markel study having a higher level of background light, as thoroughly examined in the subsequent discussion (Gagliano et al., 2020; Markel, 2020b).

\section{Are Light Conditions Prior to Experimental Treatment to Blame?}

Surprisingly, none of the participants in the ongoing discussion mentions a possibly much more important discrepancy in the light conditions during the early cultivation of seedlings between germination and the experimental treatments. While both groups used comparable irradiance (light flux per area) and photoperiod, Gagliano et al. (2016) employed cold white fluorescent light, while Markel (2020a) used illumination by blue and red LEDs "balanced for an approximation of white light" (whatever this means, possibly a blue-to-red ratio comparable with daylight). Neither of the groups provided a full description of the light sources they used, but it is clear that the spectral compositions of their lights could not have been alike.

For illustration, we are providing spectral characteristics of several light sources used in our plant culture chambers, compared to natural daylight (Fig. 1).

While none of our light sources directly corresponds to the lights used in either of the studies, we can nevertheless document that a combination of red and blue LEDs (Markel, 2020a) obviously lacked multiple spectral bands present in white fluorescent light, including wavelengths known to be perceived by plants, such as green light (Zhang et al., 2011). It is equally clear that neither of the experimental light conditions can be considered a faithful emulation of natural daylight. Additionally, as the lights 


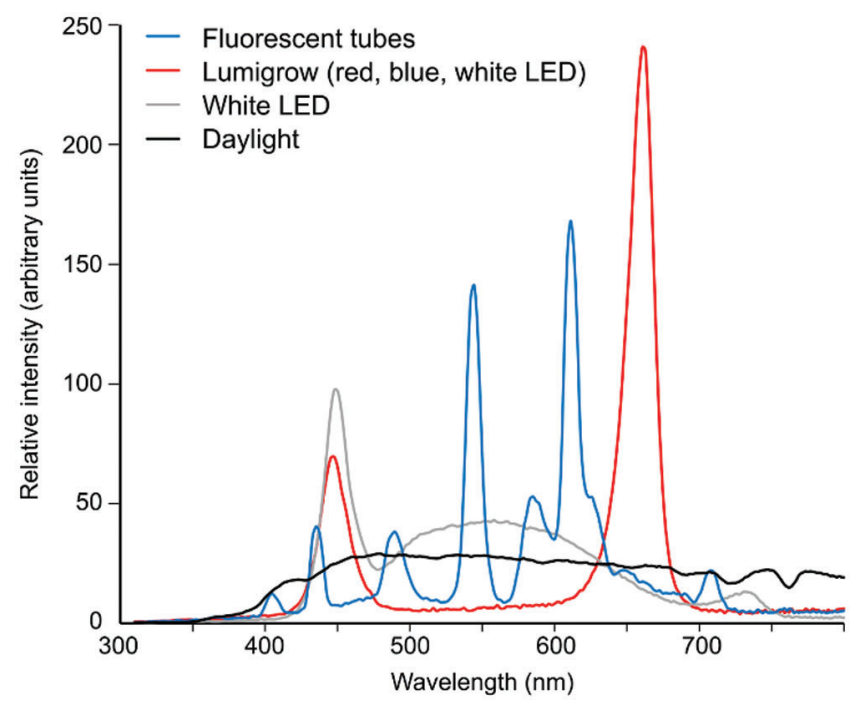

FIGURE 1. Examples of spectral profiles of several light sources commonly used for experimental plant cultivation. Light sources were: fluorescent tubes (Osram Lumilux deluxe warm white, similar to the lights used in Gagliano et al. (2016), which, however, were "cold white" and therefore apparently had a stronger blue component); a Lumigrow 325 LED panel consisting of an array of red, blue and white LEDs, with most of the light provided by the red and blue elements (similar to the lights used by Markel (2020a), except for a different blue-to-red ratio and presence of low level intermediate wavelengths); white LEDs as in the FytoScope FSWI walk-in culture chamber (Photon Systems Instruments, Drásov, Czech Republic). The Daylight plot represents early afternoon, overcast sky daylight. Light composition was measured using the SpectraPen mini spectroradiometer (Photon Systems Instruments, Drásov, Czech Republic) and normalized to equal areas under the curves in the range of $300-800 \mathrm{~nm}$.

used by the two studies differed substantially in their spectral composition, the energy available to the plants likely differed in total, as well as in particular biologically active wavelength ranges.

\section{Light History Affects Morphological and Physiological Traits}

Plants have developed complex adaptive responses to cope with variable light conditions, involving multiple organs and tissues and dependent on many genes (reviewed in Ruban (2009). A difference in the history of light quality during early growth may result in physiologically and/or morphologically different plant individuals. Indeed, spinach plants exhibit subtle but consistent developmental differences when grown under two types of white LED lights with slightly different spectral compositions (Buratini et al., 2017). In a well-characterized example, the ratio of far-red/red light, which is increased in light reflected from or filtered through leaves, initiates the "shade avoidance response"-A very complex set of structural, developmental and physiological reactions to ongoing or imminent shading. This response often includes elongation, altered flowering time, increased apical dominance and altered partitioning of resources (Ballaré and Pierik, 2017). Changes in blue-to-red light ratio also profoundly affect a plant's structure and physiological responses, and are frequently used by horticulturalists to avoid the use of growth retardants (Lykas et al., 2008) and to modulate shoot elongation in vitro (Bello-Bello et al., 2016).

Impact of a plant's light history may be long-lasting, even going beyond the individual life span. Epigenetic changes propagated by clonal as well as generative reproduction may allow offspring to pre-adapt to environments similar to those experienced by parent plants (Galloway and Etterson, 2007; Baker et al., 2019).

\section{Light Signals Contribute to Plant Memory Formation}

Light conditions play an important role in stress priming and similar plant memory phenomena (Hilker and Schmülling, 2019). Plants can modulate the activity of their photosynthetic apparatus by photoelectro-physiological signaling, processing information on past light status (Karpiński and Szechyńska-Hebda, 2010; Szechyńska-Hebda et al., 2010). In another example, a previous history of growth under monochromatic (red or blue) light decreased the photosynthetic performance of rose plants under subsequent high light conditions (Bayat et al., 2018).

Light also modulates plant responses to non-light stimuli. In Arabidopsis, salt stress induces the expression of the key proline biosynthetic enzyme (encoded by P5CS1) and the accumulation of proline. Both of these responses return to baseline upon recovery from the stress but are enhanced by recurring salt stresses, depending upon light exposure during the recovery stage. This phenomenon, which could be understood as salt-induced transcriptional memory, involves the light-dependent transcriptional regulator HY5 and light signaling-dependent maintenance of histone methylations (Feng et al., 2016). A similar mechanism may also operate during other stresses.

Other defense responses of plants, especially induction of acquired resistance and detoxification mechanisms to eliminate reactive oxygen species, are also regulated by light, including by its spectral quality prior to stress exposure (Lee et al., 2015; Han et al., 2019; Gallé et al., 2019). Several lines of evidence (reviewed in Müller-Xing et al. (2014) suggest that plants developed an epigenetic memory of UV and visible light stress. Low, ecologically relevant doses of UV-B radiation induce alterations in antioxidant status, likely protecting plants from distress when conditions worsen.

Based on such findings, we expect that prior light history affects plants' responses to adverse conditions, such as the near-total light deprivation encountered by the pea plants in the associative learning experiments (Gagliano et al., 2016; Markel, 2020a). It is quite possible that different light conditions employed in the early stages of these two studies may have contributed to their discordant outcomes. If, however, the outcome of associative learning is not robust towards growth conditions, its biological relevance may be questioned. Nevertheless, there are other phenomena that "come and go" (or teeter on the edge of statistical significance) depending on light conditions. Application of fluctuating light was, for example, recently found to elicit unexpected phenotypic variability, not observed under constant light, among Arabidopsis accessions (Kaiser et al., 2020). 


\section{Conclusions}

Given the central role of light in the life of plants, it is not surprising that the light history of an individual plant can profoundly affect not only its ontogeny and structure, but also its responses to various environmental stimuli.

Regarding the current controversy over Markel (2020a) inability to reproduce Gagliano et al. (2016)'s findings of associative learning in pea seedlings, we hypothesize that different light conditions during plant cultivation prior to the experiments may have accounted, at least in part, for the discrepancy between their results, perhaps in combination with other factors, such as the use of different plant cultivars. It thus remains to be seen whether the plant associative learning observations can be replicated under conditions identical to those employed in the original report. In the absence of additional experimental data (possibly also involving other species and different experimental setups), the question whether plants are capable of associative learning remains open.

On a more general note, the controversial case of plant associative learning should remind us of the importance of using consistent and well-documented light conditions, especially with respect to spectral quality of light, during all steps of experimental material cultivation-not only in plant memory studies but in all areas of experimental plant biology.

Acknowledgement: We thank Miloš Duchoslav (Department of Experimental Plant Biology, Faculty of Science, Charles University) for providing raw spectral measurement data for Fig. 1, Helena Lipavská (Department of Experimental Plant Biology, Faculty of Science, Charles University) for critical reading of the manuscript, and Kasey Markel and an anonymous reviewer for valuable suggestions.

Availability of Data and Materials: Data sharing is not applicable to this article as no datasets were generated or analyzed during the current study.

Author Contribution: F.C. and H.K. confirm joint responsibility for the conception of this paper, literature searches, interpretation of published data, and opinions contained in this Viewpoint. F.C. and H.K. drafted parts of the paper, F.C. produced Fig. 1 and performed final editing, F.C. and H.K. performed revision in response to reviewer feedback. Both authors approved the final version of the manuscript.

Funding Statement: This work has been supported by basic institutional funding of scientific activities at the Charles University [Progres Q43].

Conflicts of Interest: The authors declare that they have no conflicts of interest to report regarding the present study.

\section{References}

Abramson CI, Chicas-Mosier AM (2016). Learning in plants: Lessons from Mimosa pudica. Frontiers in Psychology 7: 55. DOI 10.3389/fpsyg.2016.00417.

Adelman BE (2018). On the conditioning of plants: A review of experimental evidence. Perspectives on Behavior Science 41: 431-446. DOI 10.1007/s40614-018-0173-6.
Baker BH, Sultan SE, Lopez-Ichikawa M, Waterman R (2019). Transgenerational effects of parental light environment on progeny competitive performance and lifetime fitness. Philosophical Transactions of the Royal Society B: Biological Sciences 374: 20180182. DOI 10.1098/rstb.2018.0182.

Ballaré CL, Pierik R (2017). The shade-avoidance syndrome: Multiple signals and ecological consequences. Plant, Cell \& Environment 40: 2530-2543. DOI 10.1111/pce.12914.

Bayat L, Arab M, Aliniaeifard S, Seif M, Lastochkina O, Li T (2018), Effects of growth under different light spectra on the subsequent high light tolerance in rose plants. AoB Plants 10: 161. DOI 10.1093/aobpla/ply052.

Bello-Bello JJ, Martínez-Estrada E, Caamal-Velázquez JH, MoralesRamos V (2016). Effect of LED light quality on in vitro shoot proliferation and growth of vanilla (Vanilla planifolia Andrews). African Journal of Biotechnology 15: 272-277. DOI 10.5897/AJB2015.14662.

Bhadouriya SL, Mehrotra S, Basantani MK, Loake GJ, Mehrotra R (2021). Role of chromatin architecture in plant stress responses: An update. Frontiers in Plant Science 11: 10. DOI 10.3389/fpls.2020.603380.

Bouché F, Woods DP, Amasino RM (2017). Winter memory throughout the plant kingdom: Different paths to flowering. Plant Physiology 173: 27-35. DOI 10.1104/pp.16.01322.

Buratini C, Mattoni B, Bisegna F (2017). The impact of spectral composition of white LEDs on spinach (Spinacia oleracea) growth and development. Energies 10: 1383. DOI 10.3390/ en10091383.

Burgos JE (2018). Is a nervous system necessary for learning? Perspectives on Behavior Sciences 41: 343-368. DOI 10.1007/ s40614-018-00179-7.

Calvo P, Gagliano M, Souza GM, Trewavas A (2020). Plants are intelligent, here's how. Annals of Botany 125: 11-28. DOI 10.1093/aob/mcz155.

Crisp PA, Ganguly D, Eichten SR, Borevitz JO, Pogson BJ (2016). Reconsidering plant memory: Intersections between stress recovery, RNA turnover, and epigenetics. Science Advances 2: e1501340. DOI 10.1126/sciadv.1501340.

Cvrčková F, Lipavská H, Žárský V (2009). Plant intelligence: Why, why not or where? Plant Signaling \& Behavior 4: 394-399. DOI 10.4161/psb.4.5.8276.

De la Fuente IM, Bringas C, Malaina I, Fedetz M, Carrasco-Pujante J et al. (2019). Evidence of conditioned behavior in amoebae. Nature Communications 10: a021709. DOI 10.1038/s41467-019-11677-w.

Feng XJ, Li JR, Qi SL, Lin QF, Jin JB, Hua XJ (2016). Light affects salt stress-induced transcriptional memory of P5CS1 in Arabidopsis. Proceedings of the National Academy of Sciences of the United States of America 113: E8335-E8343. DOI 10.1073/ pnas.1610670114.

Fernando CT, Liekens AM, Bingle LE, Beck C, Lenser T et al. (2009). Molecular circuits for associative learning in single-celled organisms. Journal of The Royal Society Interface 6: 463469. DOI 10.1098/rsif.2008.0344.

Gagliano M, Vyazovskiy VV, Borbély AA, Grimonprez M, Depczynski M (2016). Learning by association in plants. Scientific Reports 6: 924. DOI 10.1038/srep38427.

Gagliano M, Vyazovskiy VV, Borbély AA, Depczynski M, Radford M (2020). Comment on 'Lack of evidence for associative learning in pea plants'. eLife 9: e61141. DOI 10.7554/eLife.61141.

Gallé Á., Czékus Z, Bela K, Horváth E, Ördög A et al. (2019). Plant glutathione transferases and light. Frontiers in Plant Science 9: 474. DOI 10.3389/fpls.2018.01944. 
Galloway LF, Etterson JR (2007). Transgenerational plasticity is adaptive in the wild. Science 318: 1134-1136. DOI 10.1126/ science.1148766.

Gershman SJ, Balbi PE, Gallistel CR, Gunawardena J (2021). Reconsidering the evidence for learning in single cells. eLife 10: 1. DOI 10.7554/eLife.61907.

Han SH, Park YJ, Park CM (2019). Light priming of thermotolerance development in plants. Plant Signaling \& Behavior 14: 1554469. DOI 10.1080/15592324.2018.1554469.

Hilker M, Schmülling T (2019). Stress priming, memory, and signalling in plants. Plant, Cell \& Environment 42: 753761. DOI 10.1111/pce.13526.

Kaiser E, Walther D, Armbruster U (2020). Growth under fluctuating light reveals large trait variation in a panel of Arabidopsis accessions. Plants (Basel) 9: 316. DOI 10.3390/plants9030316.

Karpiński S, Szechyńska-Hebda M (2014). Secret life of plants: From memory to intelligence. Plant Signaling \& Behavior 5: 13911394. DOI 10.4161/psb.5.11.13243.

Lee S, Lee HJ, Jung JH, Park CM (2015). The Arabidopsis thaliana RNA-binding protein FCA regulates thermotolerance by modulating the detoxification of reactive oxygen species. New Phytologist 205: 555-569. DOI 10.1111/nph.13079.

Loshchilov I, Del Dottore E, Mazzolai B, Floreano D (2021). Conditions for the emergence of circumnutations in plant roots. PLoS One 16: e0252202. DOI 10.1371/journal.pone.0252202.

Lykas C, Kittas C, Katsoulas N, Papafotiou M (2008). Gardenia jasminoides height control using a photoselective polyethylene film. HortScience 43: 2027-2033. DOI 10.21273/HORTSCI.43.7.2027.

Lyon P (2015). The cognitive cell: Bacterial behavior reconsidered. Frontiers in Microbiology 6: 1292. DOI 10.3389/fmicb.2015.00264.

Mallatt J, Blatt MR, Draguhn A, Robinson DG, Taiz L (2021). Debunking a myth: Plant consciousness. Protoplasma 258: 459-476. DOI 10.1007/s00709-020-01579-w.

Markel K (2020a). Lack of evidence for associative learning in pea plants. eLife 9: 20. DOI 10.7554/eLife.57614.

Markel K (2020b). Response to comment on 'Lack of evidence for associative learning in pea plants'. elife 9: 835. DOI 10.7554/eLife.61689.

Mitchell A, Romano GH, Groisman B, Yona A, Dekel E et al. (2009). Adaptive prediction of environmental changes by microorganisms. Nature 460: 220-224. DOI 10.1038/ nature08112.

Müller-Xing R, Xing Q, Goodrich J (2014). Footprints of the sun: Memory of UV and light stress in plants. Frontiers in Plant Science 5: 675. DOI 10.3389/fpls.2014.00474.
Oberkofler V, Pratx L, Bäurle I (2021). Epigenetic regulation of abiotic stress memory: Maintaining the good things while they last. Current Opinion in Plant Biology 61: 102007. DOI 10.1016/j.pbi.2021.102007.

Paik I, Huq E (2019). Plant photoreceptors: Multi-functional sensory proteins and their signaling networks. Seminars in Cell \& Developmental Biology 92: 114-121. DOI 10.1016/j. semcdb.2019.03.007.

Plesser HE (2018). Reproducibility vs. replicability: A brief history of a confused terminology. Frontiers in Neuroinformatics 11: 1309. DOI 10.3389/fninf.2017.00076.

Rasmussen EB (2018). Zombies, invertebrates, and plants, Oh My! Introduction to the special section on "Learning: no brain required". Perspectives on Behavioral Science 41: 337-341. DOI 10.1007/s40614-018-00183-x.

Ruban AV (2009). Plants in light. Communicative \& Integrative Biology 2: 50-55. DOI 10.4161/cib.2.1.7504.

Sorek M, Balaban NQ, Loewenstein Y (2013). Stochasticity, bistability and the wisdom of crowds: A model for associative learning in genetic regulatory networks. PLoS Computational Biology 9: e1003179. DOI 10.1371/journal.pcbi.1003179.

Stitt M, Zeeman SC (2012). Starch turnover: Pathways, regulation and role in growth. Current Opinion in Plant Biology 15: 282-292. DOI 10.1016/j.pbi.2012.03.016.

Stolarz M (2014). Circumnutation as a visible plant action and reaction: physiological, cellular and molecular basis for circumnutations. Plant Signaling \& Behavior 4: 380-387. DOI 10.4161/psb.4.5.8293.

Szechyńska-Hebda M, Kruk J, Górecka M, Karpińska B, Karpiński S (2010). Evidence for light wavelengthspecific photoelectrophysiological signaling and memory of excess light episodes in Arabidopsis. Plant Cell 22: 2201-2218. DOI 10.1105/tpc.109.069302.

Tagkopoulos I, Liu YC, Tavazoie S (2008). Predictive behavior within microbial genetic networks. Science 320: 1313-1317. DOI 10.1126/science.1154456.

Trewavas A (2017). The foundations of plant intelligence. Interface Focus 7: 20160098. DOI 10.1098/rsfs.2016.0098.

Velez-Ramirez AI, van Ieperen W, Vreugdenhil D, van Poppel PM, Heuvelink E et al. (2014). A single locus confers tolerance to continuous light and allows substantial yield increase in tomato. Nature Communications 5: 416. DOI 10.1038/ ncomms5549.

Zhang T, Maruhnich SA, Folta KM (2011). Green light induces shade avoidance symptoms. Plant Physiology 157: 1528-1536. DOI 10.1104/pp.111.180661. 